\title{
Is the United States Country Zero for the First-World AIDS Epidemic? ${ }^{1} 23$
}

\author{
James R. Thompson \\ Dept. Statistics, Rice University \\ Houston, Texas 77251-1892 \\ email:thomp@rice.edu
}

\begin{abstract}
The incidence for AIDS per hundred thousand is several times higher in the United States than in the rest of the First World. Earlier work by Thompson indicated that a relatively small proportion of gay males frequenting the bathhouses in the United States, drove AIDS over the epidemiological threshold in the US. It is shown that the rate of growth of AIDS is the essentially the same for the United States and other First World countries. An argument is advanced, based on WHO AIDS data, to the effect that it is contact with the pool of infectives in the United States that drives the epidemic in other First World Countries.
\end{abstract}

Keywords: AIDS, bathhouses, control, international

\section{Introduction}

The AIDS epidemic began to manifest itself in the United States starting in the early 1980s. In the early days, it was almost completely a phenomenon associated with homosexual males. That such an epidemic was much more sustainable, in the First World, by male to male transmission than by heterosexual contact, should lead one to suppose that the disease was not highly infectious, that it, in fact, required virus in some quantity for transmission.

One very significant fact about the AIDS epidemic in America is that it did not start ten years earlier, when the civil restrictions against public and private homosexuality had been relaxed in the United States. This would appear to be strong prima facie evidence that gay sexual activity as practiced before 1980 would not sustain an AIDS epidemic. Furthermore, there does not seem to be evidence

\footnotetext{
${ }^{1}$ Presented at the Fifth International Conference on Population Dynamics, Zakopane, Poland, June 21-25, 1998.

${ }^{2}$ This research was supported in part by the Army Research Office (Durham) under ARODAAH04-95-1-0665.

${ }^{3}$ The author wishes to express his gratitude to Ms. Rachel Mackenzie and the other members of the Working Group on Global HIV/AIDS and STD Surveillance which is a joint Working Group between WHO and UNAIDS.
} 
that there was a significant increase in overall homosexual activity in the United States around the time of the onset of the AIDS epidemic.

Of course, this could be explained by a very long incubation period for the disease. But ten years appears very long indeed. Some conjectured that, in the First World, the United States had experienced something of a head start in whatever were the adjuvants facilitating the disease.

However, I started looking for some sociological change which might have occurred near the outbreak of the epidemic. In 1984 [2], I published a paper where it was noted that a skewing of homosexual activity to a small highly active subgroup could have the same enhancing effect as a doubling of the average number of total gay contacts in terms of driving the epidemic over the epidemiological threshold. In other words, if the average number of contacts per gay male remained constant across the gay population, then a 5\% subgroup with an activity level seven or eight times that of the $95 \%$ less active population would have the same effect as doubling the average contact rate for an entire population of homogeneously active gay males. Suppose, for example, that the contact rate of the homogeneously active gay population is $\alpha$. Then, I am maintaining the overall average contact rate at $\alpha$, which means that the contact rate for the $95 \%$ less active population drops to $\alpha / 1.35$ while the more active population rate goes to $8 \alpha / 1.35$. Given such a configuration, in spite of the fact that the total number of contacts is the same, the skewing effect is that of doubling the total number of contacts in a homogeneous activity population. The mathematical argument for this fact is available in several sources, e.g., [3], [4], [5], [6] (a brief empirical proof is given in the Appendix to this paper).

In the United States, something facilitating gay sexual activity did occur in the late 1970s, namely the de facto legalization of gay bathhouses where sex in orgiastic quantities was available without police harassment. This would assuredly be one way in which a small subpopulation of the gay community in the United States could avail themselves of activity levels well above the norm. Such establishments, interestingly enough, were more or less quickly defacilitated in other First World countries. Controlling an epidemic by defacilitating large contact rates, particularly if this can be achieved by means which are only mildly intrusive into civil liberties, would seem to be an obvious step. It was taken in Europe. Not so in the United States where bathhouses function to this day. The political machinations which caused this omission in the United States are a matter of some interest, but I shall not take the time to delve into the subject here. Suffice it to say that those persons who had the authority to set in motion the closing of the bathhouses and did not do so, may very well be responsible, however inadvertently, for causing more American deaths than those resulting from both World Wars. As of mid-1997, the total number of American AIDS cases passed 612,000. The total number of American 
HIV infectives is conservatively estimated at one million.

This emphasis on the bathhouses is of much less concern to other First World countries, where the bathhouses and their surrogates are generally not functioning. In Figure 1, we note the staggering differences in cumulative AIDS cases between the United States and France, Denmark, Netherlands, Canada, UK. The pool of infectives in the USA simply dwarfs those of the other First World countries.

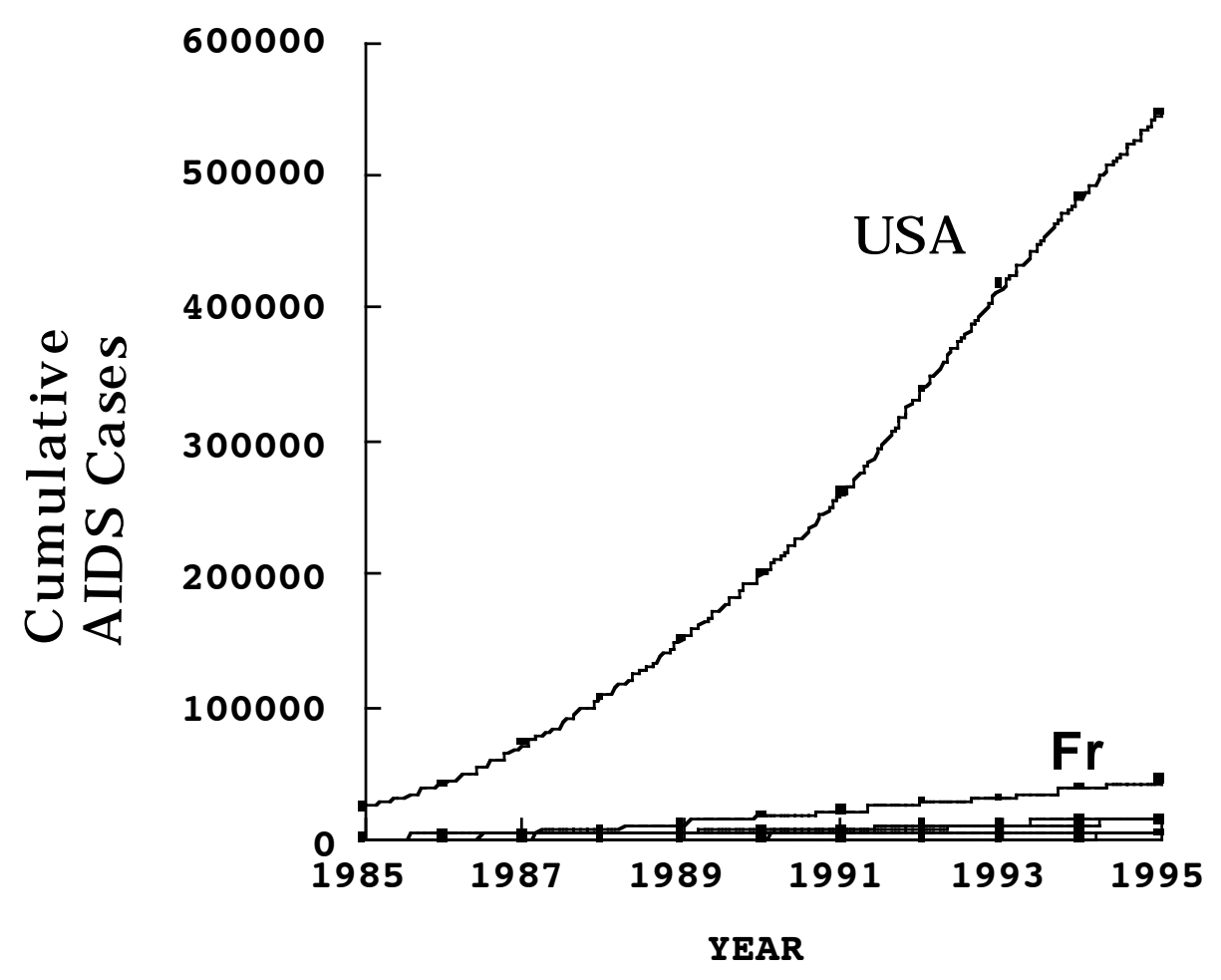

Figure 1. Cumulative AIDS Cases 1985-1995.

Shall we simply note, without undue interest that AIDS is an order of magnitude higher in the USA when compared to the average of other First World countries? I think not.

\section{Discussion}

Let us see what one might observe if other First World countries were lagging the USA. Then one would expect some sort of variation of Figure 2. 


\section{If the USA Simply Leads the Rest of the First World in ADS}

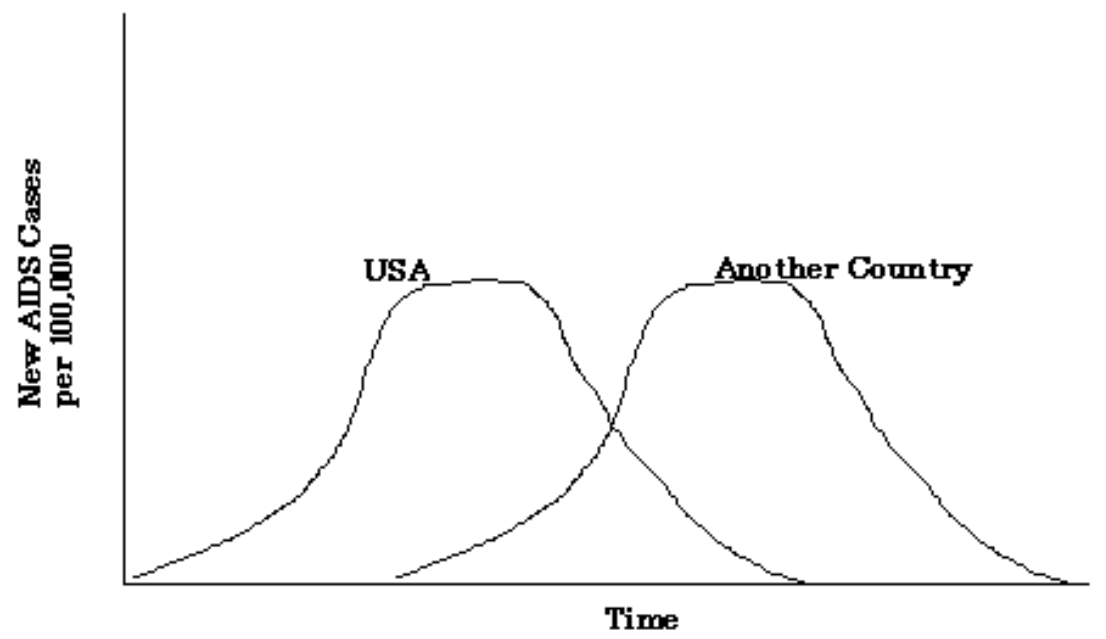

Figure 2. A Time Lagged Scenario.

That clearly is not what is happening as we see in Figure 3. No other First World country is catching up to the USA. Moreover, a downturn in new case rates is observable in all the countries shown.

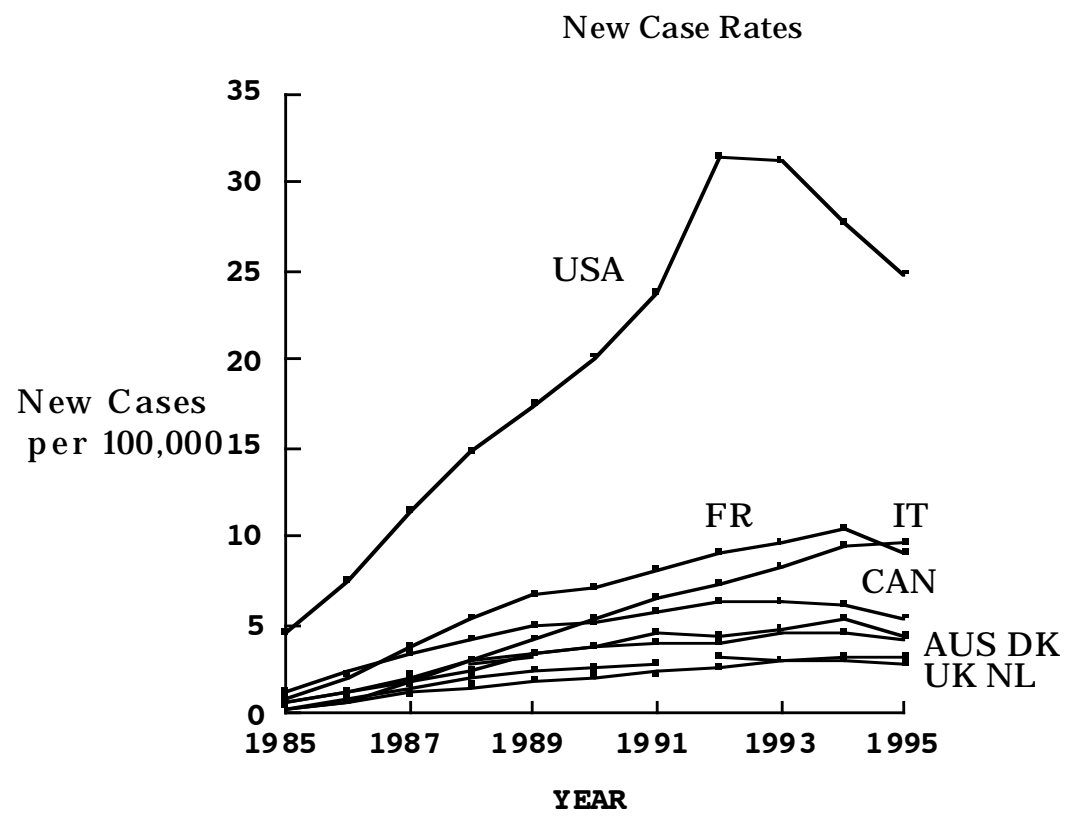

Figure 3. New Case Rates per 100,000. 
Further insight is given in Figure 4 where we divide the annual incidence of AIDS per 100,000 in the USA by that for various other First World countries.

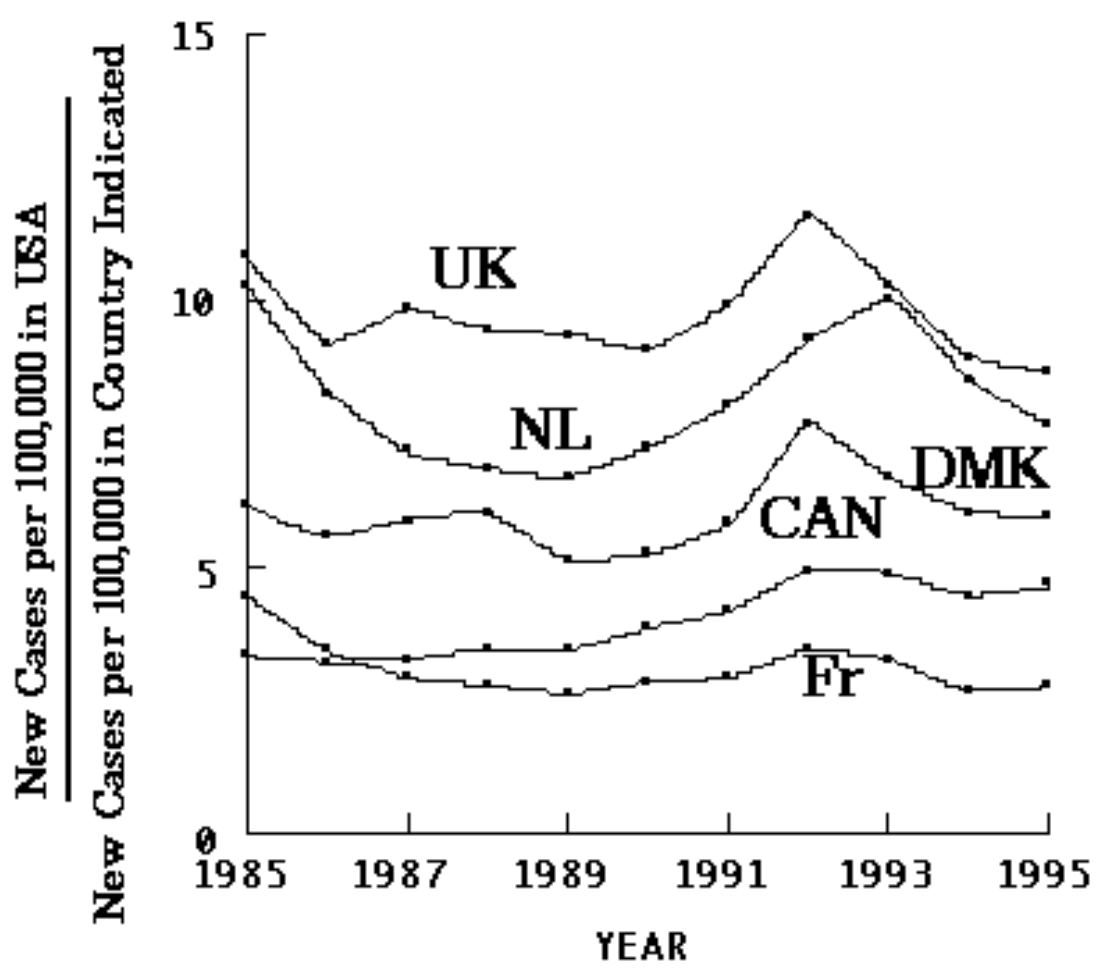

Figure 4. Comparative New Case Rates.

There seems to be a relative constancy of the new case ratio across the years for each country when compared to the USA. Thus, for the United Kingdom, it is around 9 , for Denmark 6 , etc. It is a matter of note that this relative constancy of new case rates is maintained over the period examined (eleven years).

We notice, in Figure 5, that the cumulative instance per 100,000 of AIDS in the USA divided by that for other First World countries gives essentially the same values as shown for the new case rates in Figure 4. 


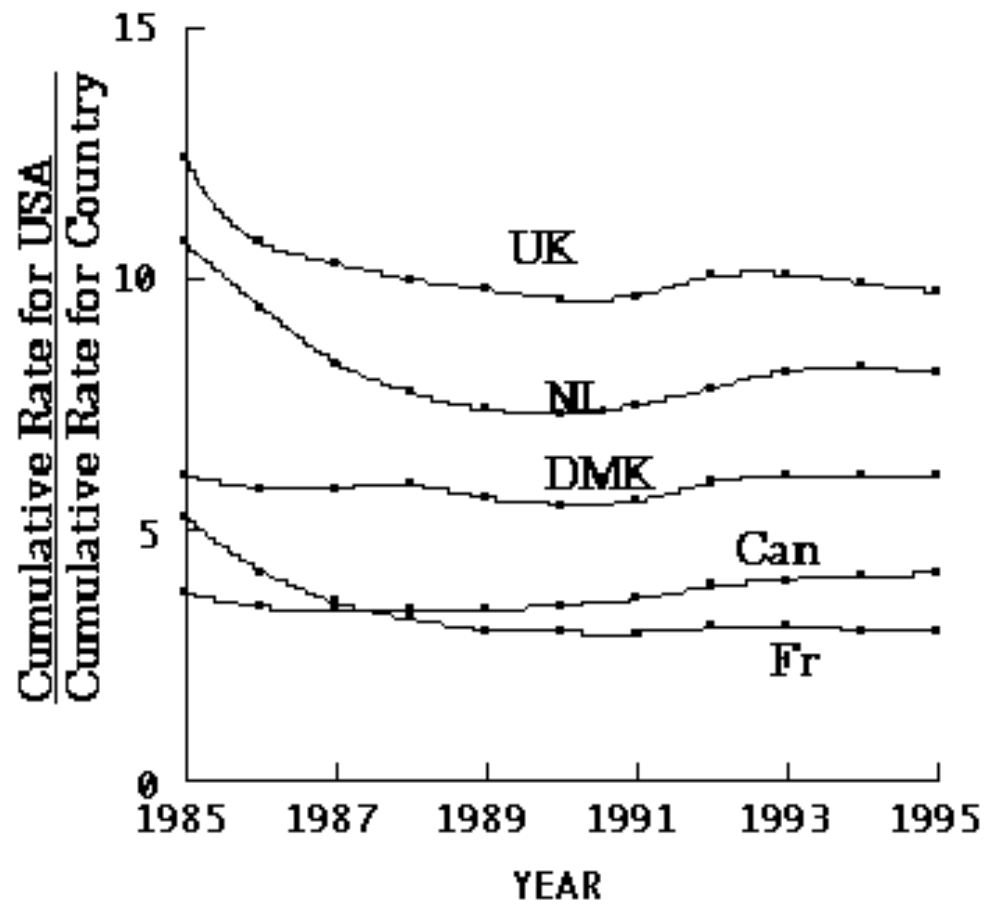

Figure 5. Comparative Cumulative Case Incidence.

Next, let us consider the piecewise in time exponential model for the number of AIDS cases in, say, Country A.

$$
\frac{d y_{A}}{d t}=k_{A}(t) y_{A}
$$

We show in Figure 6 estimates for $k$ rates on a year by year basis using

$$
k_{A}(t) \approx \frac{\text { new cases per year }}{\text { cumulative cases }}
$$




\section{Rates for Canada, Denmark, France, Netherlands, United Kingdom, United States}

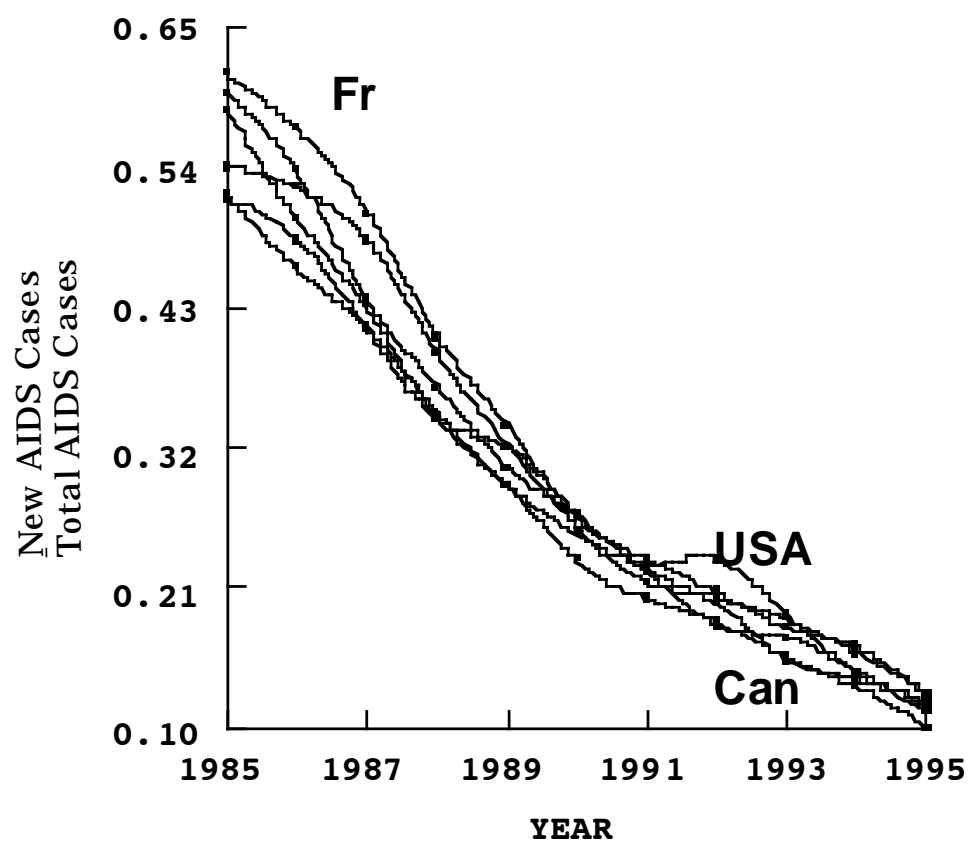

Figure 6. $k_{\text {Country }}(t)$ Values.

We note the apparent near equality of rates for the countries considered. To show this more clearly, in Figure 7, we show the ratio of the annual estimated piecewise national rates divided by the annual estimated rate of the USA.

It is a matter of some interest that $k$ values are essentially the same for each of the countries shown in any given year. How shall we explain a situation where one country has a much higher incidence of new cases, year by year, and the rate of increase for all the countries is the same? For example, by mid 1997, the United Kingdom had a cumulative total of 15,081 cases compared to the United States' 612,078 . This ratio is 40.59 whereas the ratio of populations is only 4.33 . This gives us a comparative incidence proportion of 9.37. On the other hand, at the same time, Canada had cumulative AIDS total of 15,101 . The US population is 9.27 times that of Canada. The comparative incidence proportion for the USA versus Canada in mid-1997 was 4.37. The comparative incidence of the USA vis-a-vis the UK is over twice that of the USA vis-a-vis Canada. Yet, in all three countries the rate of growth 
of AIDS cases is nearly the same. This rate changes from year to year, from around .54 in 1985 to roughly .12 in 1995 . Yet it is very nearly the same for each country in any given year. One could, therefore, predict the number of new cases in France, in a given year, just about as well knowing the case history of the United States instead of that in France. The correlation of new cases for the United States with that for each of the other countries considered is extremely high, generally around .96. Can we explain this by an appeal to some sort of magical synchronicity? I think not. Particularly since we have the fact that though the growth rates of AIDS for the countries are roughly the same for any given year, the new case relative incidence per 100,000 for the United States is several times that of any of the other countries.

Rates Relati ue to USA

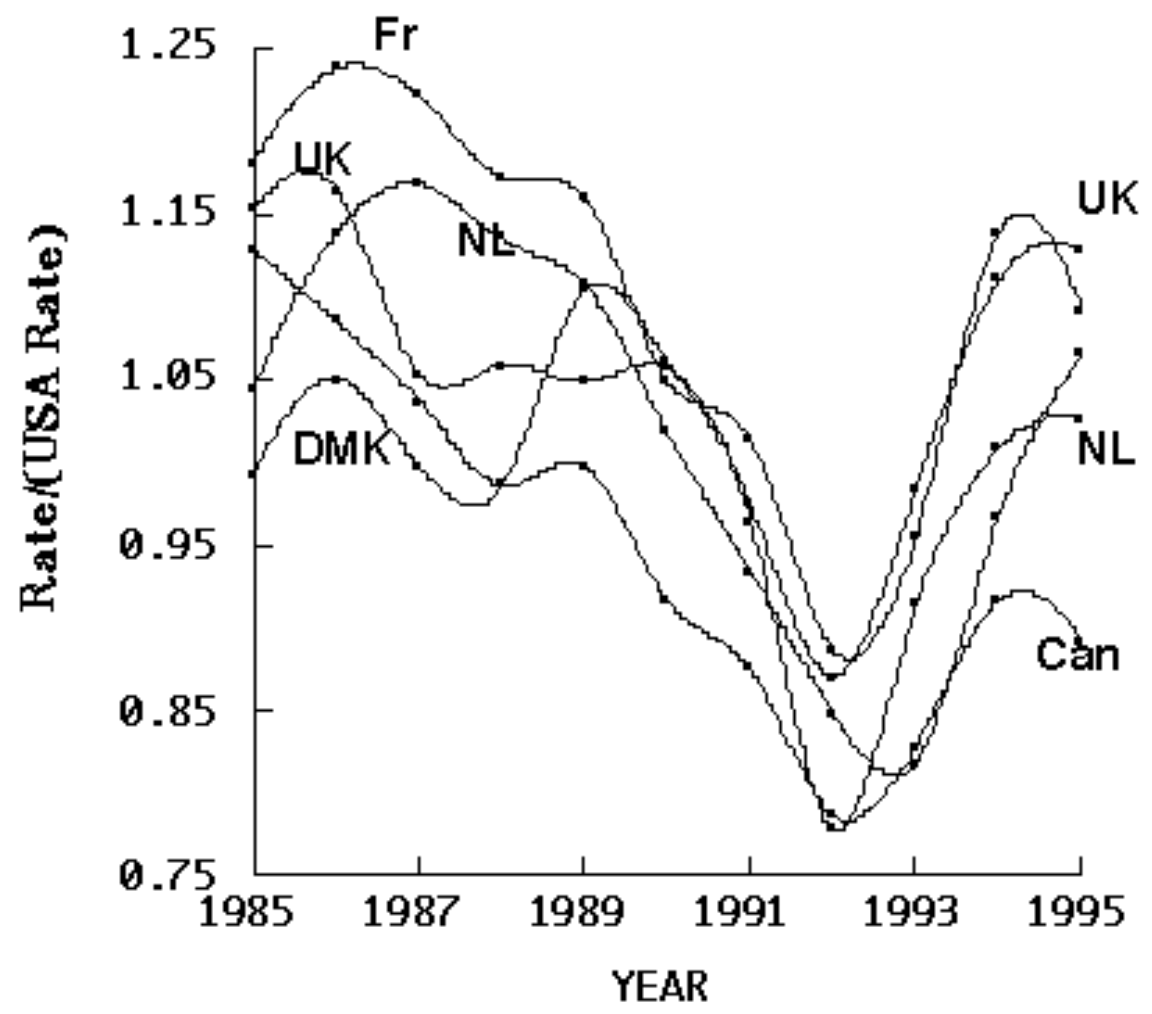

Figure 7. Ratios of Piecewise Rate Estimates.

Earlier in this paper we noted the conjecture I made in the mid-80s that it was the bathhouses which caused the stand-alone epidemic in the United States. But, 
as we have seen, the bathhouse phenomenon really does not exist in the rest of the First World. How is it, then, that there are stand-alone AIDS epidemics in each of these countries? I do not believe there are stand-alone AIDS epidemics in these countries.

In Figure 8, let us suppose there is a country, say Country Zero, a country where, for whatever reason, the sociology favors a standalone AIDS epidemic. From other First World countries there is extensive travel to and from Country Zero. If AIDS, with its a very low infectivity rates breaks out in Country Zero, then, naturally, the disease will spread to the other countries. But, if the infectivity level is sufficiently low, then the maintenance of an apparent epidemic in each of the countries will be dependent on continuing visits to and from Country Zero.

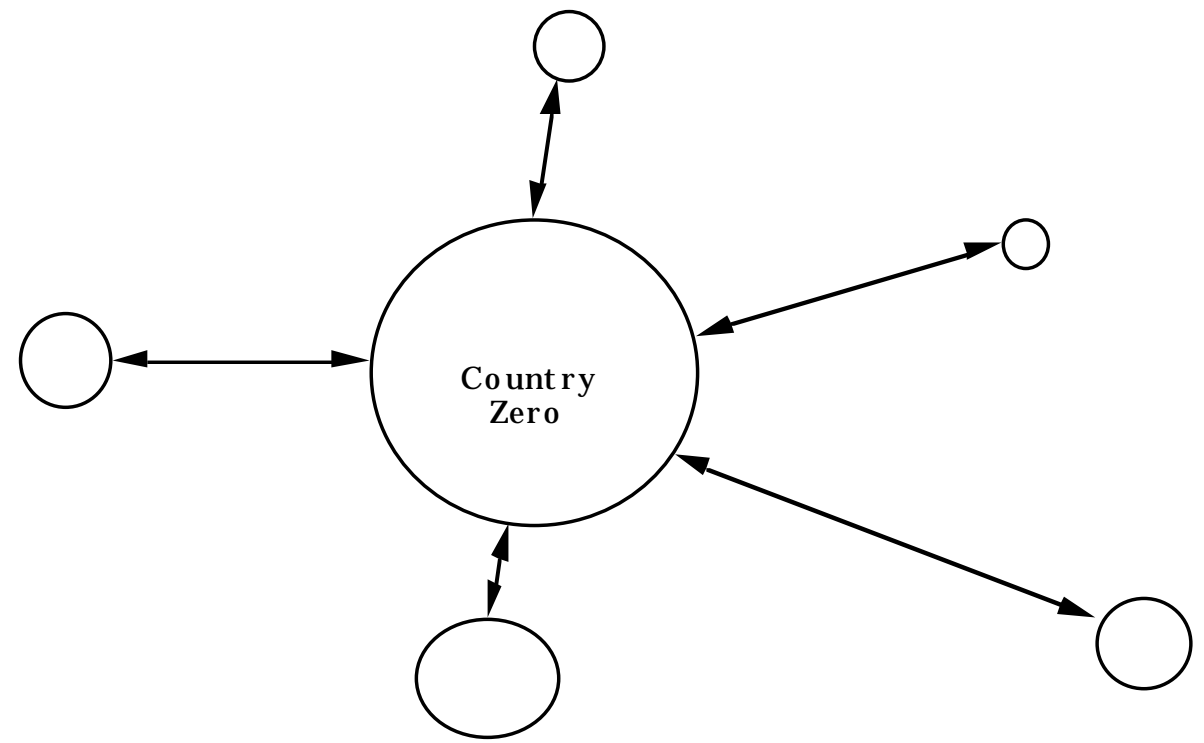

Figure 8. Country Zero.

Now let us suppose the fraction of infectives is rather low in country $j$. Thus, we shall assume that the susceptible pool is roughly constant. Let the number of infectives in country $j$ be $x_{j}$. Let the number of infectives in Country Zero be given by $z$. Suppose the case rate in Country Zero divided by that for country $j$ is relatively constant, say $c_{j}$. Let us suppose we have the empirical fact that, both for Country Zero and the other countries, we can use the same $\beta_{t}$ in

$$
\begin{aligned}
\frac{d z}{d t} & =\beta_{t} z \\
\frac{d x_{j}}{d t} & =\beta_{t} x_{j}
\end{aligned}
$$


Let us suppose that, at any given time, the transmission of the disease in a country is proportional both to infectives in the country and to infectives in Country Zero. Let us suppose that the population of infectives in country $j$ is given by $x_{j}$ and in Country $j$ is $z$. Let the population of country $j$ be given by $N_{j}$ and that of Country Zero be given by $N_{Z}$. Suppose that we have, at any time, the following constancy

$$
\frac{z / N_{Z}}{x_{j} / N_{j}}=c_{j}
$$

Then we have

$$
\begin{aligned}
\frac{d x_{j}}{d t} & =\alpha_{j, t} x_{j}+\eta_{j, t} z \\
& =\left(\alpha_{j, t}+\frac{N_{Z}}{N_{j}} c_{j} \eta_{j, t}\right) x_{j} \\
& =\beta_{t} x_{j}
\end{aligned}
$$

where $\alpha_{j, t}$ and $\eta_{j, t}$ are the transmission rates into country $j$ from the country infectives and the Country Zero infectives, respectively. Now, we are assuming that the effect of infectives from the countries will have relatively little effect on the increase of infectives in Country Zero. Thus, for a short time span,

$$
z(t) \approx z(0) e^{\beta_{t} t}
$$

Thus (6) is roughly

$$
\frac{d x_{j}}{d t}=\alpha_{j, t} x_{j}+\eta_{j, t} z(0) e^{\beta_{t} t}
$$

Now, we note that the epidemic in a country can be sustained even if $\alpha_{j, t}$ is negative, provided the transmission from the Country Zero infectives is sufficiently high. If we wish to look at the comparative effect of Country Zero transmission on country $j$ vis-a-vis country $i$, we have

$$
\eta_{j, t}=\frac{c_{i}}{c_{j}} \frac{N_{j}}{N_{i}} \eta_{i, t}+\frac{\alpha_{i, t}-\alpha_{j, t}}{c_{j}} \frac{N_{j}}{N_{i}}
$$

Let us suppose that for two countries, we have

$$
\alpha_{j, t}=\alpha_{i, t}
$$

Then we have

$$
\eta_{j, t}=\frac{c_{i}}{c_{j}} \frac{N_{j}}{N_{i}} \eta_{i, t}
$$


Looking at this another way, we have

$$
\frac{x_{j}}{x_{i}}=\frac{\eta_{j}}{\eta_{i}}
$$

If $\eta_{j}$ doubles, then according to the model, the number of infectives in country $j$ doubles. Let us see what the situation would be in Canada if, as a standalone, the epidemic is just at the edge of sustainability, i.e.,

$$
\alpha_{C a n}=0
$$

Then, going back to a universal $\beta_{t}$ for all the countries and Country Zero America as well, we have

$$
\begin{aligned}
\eta_{C a n, t} & =\frac{N_{C a n}}{N_{U S A}} \frac{1}{c_{C a n}} \beta_{t} \\
& =\frac{26,832,000}{248,709,873} \frac{1}{4.14} \beta_{t} \\
& =.026 \beta_{t}
\end{aligned}
$$

Thus, according to the model, activity rates from USA infectives roughly $2.6 \%$ that experienced in the USA could sustain a Canadian epidemic at a comparative incidence ratio of around 4 to 1, US to Canadian. (If someone would conjecture that it is rather the Canadian infectives that are causing the epidemic in the United States, that would require that the activity rate of Canadian infectives with American susceptibles must be $1 / .026=38.5$ times that of Canadian infectives with Canadian susceptibles.) If this activity would double to $5.2 \%$, then the Canadian total infectives would double, but the rate $\left(1 / x_{C a n}\right) d x_{C a n} / d t$ would still grow at rate $\beta_{t}$.

Similarly, we can show that

$$
\begin{aligned}
\eta_{F r, t} & =.076 \beta_{t} \\
\eta_{U K, t} & =.024 \beta_{t} \\
\eta_{D k, t} & =.0034 \beta_{t} \\
\eta_{N L, t} & =.0075 \beta_{t}
\end{aligned}
$$

\section{Conclusions}

We have observed some surprises and tried to come up with plausible explanations for those surprises. The relative incidence of AIDS for various First World countries when compared to that of the United States appears, for each country, to be 
relatively constant over time and this incidence appears to be roughly the same for cumulative ratios and for ratios of new cases. We have seen that one $k_{\text {Country }}(t)=$ $\beta_{t}$ may be used. The rate of growth for AIDS, $\beta_{t}$, changes year by year, but it seems to be nearly the same for the all the First World countries considered (Figure 6), including the USA. The bathhouse phenomenon which I have repeatedly argued as an essential for the maintenance of a standalone epidemic of AIDs in a First World country is generally not present in First World countries other than the United States. Yet AIDS has a continuing small (compared to that of the USA), though significant, presence in First World Countries other than the United States. The new case (piecewise exponential) rate there tracks that of the United States rather closely, country by country. We have shown that a model where a term for "travel" from and to the USA is dominant does show one way in which one can explain the surprises. Some years ago [2], I pointed out that the American gay community was made unsafe by the presence of a small subpopulation which visited the bathhouses, even though the large majority of gays, as individuals, might not frequent these establishments. The present work gives some indication that the high AIDS incidence in the United States should be a matter of concern to other First World countries as long as travel to and from the USA continues at the brisk rates we have seen since the early 1980s.

Nothing could have been easier than American officials closing down the bathhouses in the early 1980's, an action taken quickly by the French and by other First World countries who had bathhouses or bathhouse surrogates. That the American officials did not take similar steps has caused the epidemic to rage as a standalone in the United States. There is some acknowledgment within the leadership of the American gay community of the effect of the small subgroup of high sexual activity in pushing AIDS from endemic to epidemic in the United States [1].

At the time of the publication of [2] in 1984, there was little motion on the part of American public health officials to shutting down the bathhouses and their surrogates. (In fact, they are still not closed.) It was also clear that the Europeans were shutting them down as a matter of course. The results of these varying policies now seem rather clear. The American AIDS epidemic rages as a standalone with expected fatalities in excess of one million. The Europeans have rates which are an order of magnitude less than those in the United States. Yet, as we have seen, their epidemics appear not to be standalone, but rather due to contacts with the huge pool of American infectives.

The failure of American public health officials to take the simple step of shutting down the gay bathhouses will cost the United States more dead citizens than all the wars of the Twentieth Century. There is the added humiliation that the USA is essentially a Typhoid Mary country, causing AIDS epidemics in other First World 
countries. The drama of the mismanagement of the American AIDS epidemic continues. And, like a Greek tragedy, it proceeds painfully and incrementally towards an impending doom.

\section{Appendix}

Let us consider the situation where there are two gay populations, the majority, less sexually active, and a minority (e.g., bathhouse visitors) with greater activity than that of the majority. In the following, we shall use the subscript "1" to denote the majority, less sexually active portion of the target (gay) population, and the subscript " 2 " to denote the minority, sexually very active portion (the part which engages in high frequency anonymous anal intercourse, typically at bathhouses). The more active population will be taken to have a contact rate $\tau$ times that of the rate $k$ of the majority portion of the target population. The fraction of the more sexually active population will be taken to be $p$.

$$
\begin{aligned}
\frac{d Y_{1}}{d t} & =\frac{k \alpha X_{1}\left(Y_{1}+\tau Y_{2}\right)}{X_{1}+Y_{1}+\tau\left(Y_{2}+X_{2}\right)}-(\gamma+\mu) Y_{1} ; \\
\frac{d Y_{2}}{d t} & =\frac{k \alpha \tau X_{2}\left(Y_{1}+\tau Y_{2}\right)}{X_{1}+Y_{1}+\tau\left(Y_{2}+X_{2}\right)}-(\gamma+\mu) Y_{2} ; \\
\frac{d X_{1}}{d t} & =-\frac{k \alpha X_{1}\left(Y_{1}+\tau Y_{2}\right)}{X_{1}+Y_{1}+\tau\left(Y_{2}+X_{2}\right)}+(1-p) \lambda-\mu X_{1} ; \\
\frac{d X_{2}}{d t} & =-\frac{k \alpha \tau X_{2}\left(Y_{1}+\tau Y_{2}\right)}{X_{1}+Y_{1}+\tau\left(Y_{2}+X_{2}\right)}+p \lambda-\mu X_{2} .
\end{aligned}
$$

where

$k=$ number of contacts per month;

$\alpha=$ probability of contact causing AIDS;

$\lambda=$ immigration rate into sexually active gay population;

$\mu=$ emigration rate from sexually active gay population;

$\gamma=$ marginal emigration rate from sexually active gay population due

to sickness and death;

$X=$ number of susceptibles;

$Y=$ number of infectives.

We note that even with a simplified model such as that presented here, we appear to be hopelessly overparameterized. There is little chance that we shall have reliable estimates of all of: $k, \alpha, \gamma, \mu, \lambda, p, \tau$. One of the techniques sometimes available to the 
modeller is to express the problem in such a form that most of the parameters will cancel. For the present case, we will attempt to determine the $k \alpha$ value necessary to sustain the epidemic for the heterogeneous case when the number of infectives is very small.

If $Y_{1}=Y_{2}=0$, then the equilibrium values for $X_{1}$ and $X_{2}$ are $(1-p)(\lambda / \mu)$ and $p(\lambda / \mu)$, respectively. Expanding the right-hand sides of (15) in a Maclaurin series, we have (using lower case symbols for the perturbations from 0 ),

$$
\begin{aligned}
\frac{d y_{1}}{d t} & =\left[\frac{k \alpha(1-p)}{1-p+\tau p}-(\gamma+\mu)\right] y_{1}+\frac{k \alpha(1-p)}{1-p+\tau p} y_{2} \\
\frac{d y_{2}}{d t} & =\frac{k \alpha \tau p}{1-p+\tau p} y_{1}+\left[\frac{k \alpha \tau^{2} p}{1-p+\tau p}-(\gamma+\mu)\right] y_{2} .
\end{aligned}
$$

Summing, we have

$$
\frac{d y_{1}}{d t}+\frac{d y_{2}}{d t}=\left[\frac{k \alpha(1-p)+k \alpha \tau}{1-p+\tau p}-(\gamma+\mu)\right] y_{1}+\left[\frac{k \alpha \tau^{2} p+k \alpha(1-p)}{1-p+\tau p}-(\gamma+\mu)\right] y_{2}
$$

In order for the coefficient of $y_{2}$ to be negative, we require:

$$
k \alpha<(\gamma+\mu)\left[\frac{1-p+\tau p}{\tau^{2} p+1-p}\right]=k^{*} \alpha
$$

In order for the coefficient of $y_{1}$ to be negative, we require

$$
k \alpha<(\gamma+\mu)
$$

Since $\tau \geq 1$, the condition in (18) will guarantee that the inequality in (19) will be satisfied as well. Now, in the homogeneous contact case (i.e., $\tau=1$ ), we note that for the epidemic not to be sustained we require the condition in equation (20), i.e.,

$$
k_{H} \alpha<(\gamma+\mu)
$$

For the heterogeneous contact case with $k^{*}$, the average contact rate is given by

$$
\begin{aligned}
k_{\text {ave }} \alpha & =p \tau\left(k^{*} \alpha\right)+(1-p)\left(k^{*} \alpha\right) \\
& =\frac{[p \tau+(1-p)]^{2}}{\tau^{2} p+1-p}
\end{aligned}
$$

So, dividing the sustaining $k_{H} \alpha$ by the sustaining value for the heterogeneous contact rate, we have

$$
Q=\frac{1-p+\tau^{2} p}{(1-p+\tau p)^{2}}
$$


When we have a subpopulation of fraction .05 whose activity is eight times that of the majority, less active, population, this gives a $Q$ value of 2.28 . Thus, keeping the total number of contacts constant, moving from a homogeneous activity target population to the heterogeneous population described, has the same effect in driving the endemic to an epidemic as if everyone in the homogeneous population had more than doubled his rate of contact.

For a fixed value of $\tau$, we have that $Q$ is maximized when

$$
p=\frac{1}{1+\tau}
$$

For this value of $p$, we have

$$
Q=\frac{(1+\tau)^{2}}{4 \tau}
$$

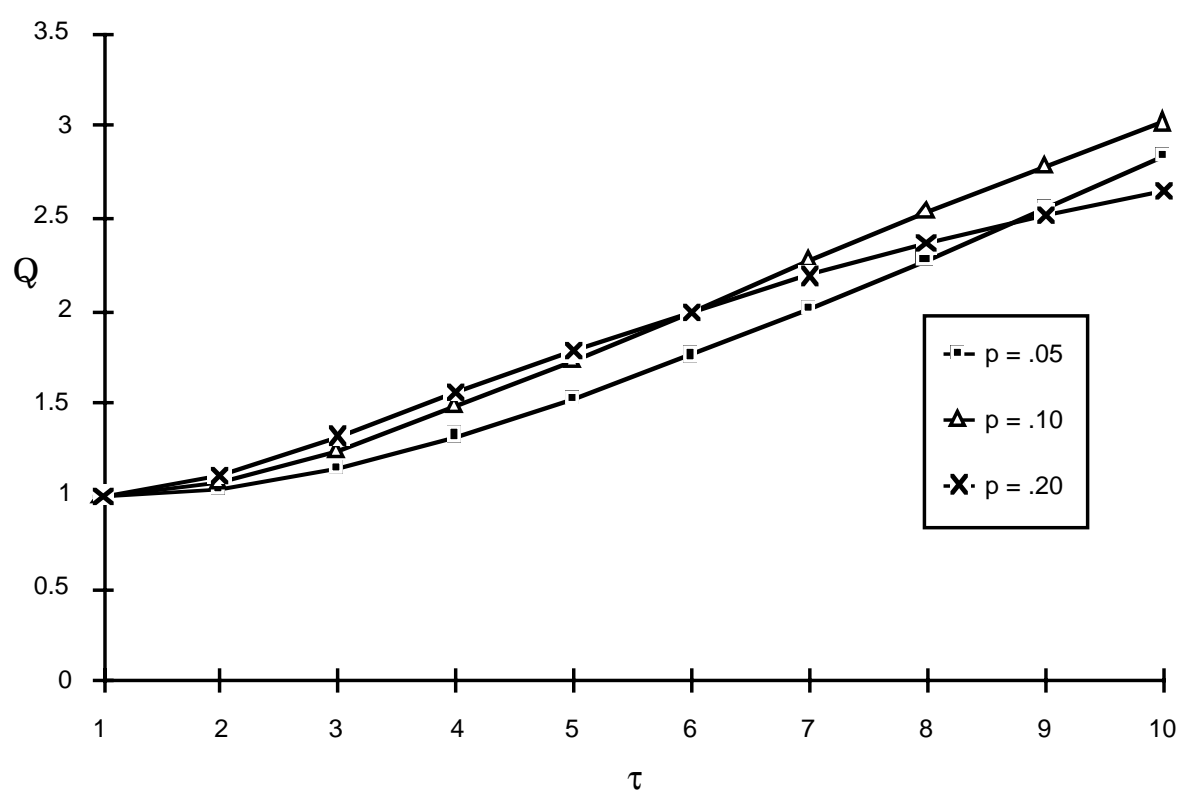

Figure 9. Effect Of High Activity Subpopulation 


\section{References}

[1] Rotello, Gabriel (1997). Sexual Ecology: AIDS and the Destiny of Gay Men. New York: Dutton, pp. 85-89.

[2] Thompson, James R. (1984) "Deterministic versus stochastic modeling in neoplasia" Proceedings of the 1984 Computer Simulation Conference, New York: North Holland, pp. 822-825.

[3] Thompson, James R. (1989) Empirical Model Building. New York: John Wiley \& Sons, pp. 79-91.

[4] Thompson, James R. and Tapia, Richard A. (1990) Nonparametric Function Estimation, Modeling and Simulation, Philadelphia: SIAM, pp. 233-243.

[5] Thompson, James R. (1989) "AIDS: the mismanagement of an epidemic," Computers Math. Applic., v. 18, pp. 965-972.

[6] Thompson, James R.(1998) "The United States AIDS Epidemic in First World Context," (1998) in Advances in Mathematical Population Dynamics: Molecules, Cells and Man,Arino, Axelrod and Kimmel, eds., World Scientific Publishing Company, pp. 345-354.

[7] West, R. Webster (1994). Modeling the Potential Impact of HIV on the Spread of Tuberculosis in the United States, doctoral dissertation, Rice University

[8] West, R. Webster and Thompson, James R. "Modeling the impact of HIV on the spread of tuberculosis in the United States," (1998) in Mathematical Biosciences 143:35-60.

[9] West, R. Webster and Thompson, James R. "Models for the Simple Epidemic" (1998) in Mathematical Biosciences 141:29-39. 